\title{
„Man gesteht sich ja heutzutage keine Depression mehr ein, sondern nennt es Burnout“"
}

\author{
Berichterstattung über Burnout in deutschen Printmedien \\ “Nowadays, we wouldn't Admit Being Depressed - Instead, \\ we Tend to Call it Burnout"
}

Reporting on Burnout in German Print Media

Autoren

Theresia Rechenberg ", Julia Nowikow*, Georg Schomerus

Institut

Klinik und Poliklinik für Psychiatrie und Psychotherapie, Universitätsklinikum Leipzig

Schlüsselwörter

Burnout, Depression, Medien

Keywords

burnout, depression, media

Online-Publikation 8.4 .2020

Bibliografie

Psychiat Prax 2020; 47: 426-432

DOI 10.1055/a-1142-9140

ISSN 0303-4259

(C) 2020. Thieme. All rights reserved.

Georg Thieme Verlag KG, Rüdigerstraße 14,

70469 Stuttgart, Germany

\section{Korrespondenzadresse}

Theresia Rechenberg, M.A., Universitätsklinikum Leipzig, Klinik und Poliklinik für Psychiatrie und Psychotherapie, Semmelweisstraße 10, Haus 13, 04103 Leipzig, Deutschland Theresia.Rechenberg@medizin.uni-leipzig.de

\section{ZUSAMMENFASSUNG}

Ziel der Studie Identifizierung von Überschneidungen und Differenzen zum Störungsbild Depression, die sich in der Berichterstattung über Burnout zeigen.

Methodik Qualitative Inhaltsanalyse von 3 überregionalen Tageszeitungen aus den Jahren 2016 und 2017.
Ergebnisse Häufig weist die dargestellte Symptomatik von Burnout Bezüge zu den Leitsymptomen einer Depression auf. Differenzen werden in den dargestellten Ursachen und therapeutischen Maßnahmen sichtbar.

Schlussfolgerung In der Berichterstattung erscheint das Phänomen Burnout durch den Arbeitsbezug, die bildliche Qualität des Begriffs und die zahlreichen Offenlegungen prominenter Betroffener nahbarer als eine Depression. Vor- und Nachteile dieses Phänomens werden im Hinblick auf Stigmatisierung diskutiert.

\section{ABSTRACT}

Objective Identifying overlaps with and differences to the clinical picture of depression as reflected in media coverage of burnout.

Methods Qualitative content analysis of three national daily newspapers from the years 2016 and 2017.

Results Depictions of burnout symptoms are often related to the leading symptoms of depression. Differences become visible in the underlying causes and therapeutic measures described. Burnout is rarely depicted in the context of professional treatment recommendations.

Conclusion In coverage, the phenomenon of burnout seems more approachable than depression due to its work-relatedness, the figurative quality of the term, and numerous disclosures by affected celebrities. We discuss that the destigmatizing potential of the term burnout may come at the expense of people with severe depression, and people outside the working context. Additionally, lower recommendation of professional treatment for burnout may delay help-seeking.

\footnotetext{
* geteilte Erstautorenschaft
} 


\section{Einleitung}

Im Juni 2018 hat die Weltgesundheitsorganisation (WHO) die 11. Revision der Internationalen Klassifikation der Krankheiten ICD-11 vorgestellt, die ab Januar 2022 in Kraft treten soll und nun auch Störungen klassifiziert, die bislang noch nicht als solche anerkannt wurden [1]. Schenkt man einer nicht unerheblichen Anzahl von Medien - darunter auch Onlineformate populärer Tageszeitungen - Glauben, erkennt die WHO nun auch Burnout als Krankheit an [2-4]. Dass es sich dabei um eine Fehlinterpretation und damit gleichzeitig um eine Falschmeldung handelt, ahnen viele Leser nicht. In der ICD-11 wird Burnout als Syndrom beschrieben, das aus chronischem Stress am Arbeitsplatz hervorgeht, der noch nicht erfolgreich bewältigt wurde [5]. Damit gilt Burnout wie auch schon in der ICD-10 lediglich als Faktor, der den Gesundheitszustand beeinflusst und somit nicht als Krankheit [5].

Die Rolle, die den Massenmedien als Informationsquelle bei der Vermittlung von Wissen und Bildung von Einstellungen zukommt, ist groß [6, 7]. Das zeigt nicht nur das direkte Medienecho auf die Revision der ICD, sondern auch die Tatsache, wie viele Stellungnahmen [8,9] und Richtigstellungen [10-13] zu den Falschmeldungen zu finden sind. Die Medien bilden mit ihrer Verunsicherung auch ab, was die Wissenschaft zum Thema Burnout beschäftigt: die Suche nach Souveränität und Konsens in Bezug auf den Umgang mit dem Phänomen Burnout. Denn bislang fehlen sowohl eine einheitliche Definition von Burnout [14] als auch Erklärungsansätze oder geeignete Diagnoseinstrumente und -kriterien $[15,16]$.

Seitdem der Psychoanalytiker Herbert J. Freudenberger mit einer Veröffentlichung im Jahr 1974 den Begriff Burnout popularisierte, wurden zahlreiche Definitionsversuche unternommen [17], aus denen Schaufeli und Enzmann [18] wesentliche Aussagen in folgender Definition zusammenfassen: „Burnout ist ein dauerhafter, negativer, arbeitsbezogener Seelenzustand ,normaler ' Individuen. Er ist in erster Linie von Erschöpfung gekennzeichnet, begleitet von Unruhe und Anspannung (distress), einem Gefühl verringerter Effektivität, gesunkener Motivation und der Entwicklung dysfunktionaler Einstellungen und Verhaltensweisen bei der Arbeit. Diese psychische Verfassung entwickelt sich nach und nach, kann dem betroffenen Menschen aber lange unbemerkt bleiben. Sie resultiert aus einer Fehlpassung von Intentionen und Berufsrealität.“ Die hier anklingenden Symptome von Burnout weisen große Ähnlichkeit zu den Leitsymptomen einer Depression auf, die mit gedrückter Grundstimmung, Interessenverlust und Freudlosigkeit sowie Antriebsminderung und erhöhter Ermüdbarkeit beschrieben werden [19]. Bei Burnout lässt sich jedoch sowohl in Übereinstimmung mit der WHO und der Deutschen Gesellschaft für Psychiatrie, Psychotherapie und Nervenheilkunde (DGPPN) als auch in einem weiteren Definitionsversuch von Maslach (1982) ein starker Bezug zu Arbeitsüberforderung erkennen. Damit korrespondieren auch Befunde von Bahlmann et al. [20, 21] aus einer 2011 realisierten repräsentativen Bevölkerungsumfrage, in der den Befragten eine Fallvignette vorgelegt wurde, die unter anderem eine Person mit einer depressiven Episode beschrieb, ohne dass die Diagnose benannt wurde [22]. 10,2\% der Befragten bezeichneten den Zustand der beschriebenen Person als Burnout, wovon ein Großteil (74\%) den Zustand auch als Krankheit im medizinischen Sinne einschätzte [20]. Nur wenig mehr (77\%) von denjenigen Befragten, die in dem Problem eine Depression sahen, bezeichneten diese ebenfalls als Krankheit [20]. Der Wunsch nach sozialer Distanz war bei den Befragten, die den Zustand der beschriebenen Person als Burnout wahrnahmen, geringer als bei den Befragten, die darin eine Depression sahen [20].

Doch wie erschließen sich Menschen ihr Bild von Burnout und Depression durch die Medien? Einerseits fungieren Medien als Spiegel der Gesellschaft, der zeigt, wie sich psychische Störungen darin aktuell äußern, welche Erklärungsmodelle diese Gesellschaft für ihre Entstehung anbietet und welche Möglichkeiten sie zu ihrer Behandlung hat [23]. Andererseits bieten insbesondere Massenmedien nicht nur eine Bestandsaufnahme von Phänomenen, sondern können auf vielfältige Weise Einfluss auf Meinungsbildungsprozesse nehmen [24]: Sie können Wissen vermitteln, eine Agenda setzen und bestimmte Aspekte oder Interpretationen eines Phänomens hervorheben (Framing). Indem Medien ein Meinungsklima darstellen, können sie eine Brücke zwischen individueller und öffentlicher Meinungsbildung schlagen. Durch die direkte und indirekte Beeinflussung der Einstellungen von Rezipienten zu bestimmten Fragestellungen, können Medien auch eine Persuasionsfunktion einnehmen. Am Ende einer möglichen Wirkkette der Medien stehen deren Anregungen zu konkretem Handeln. Da psychische Krankheiten häufig unbehandelt bleiben und nach wie vor tabuisiert werden, ist eine verantwortliche, sachlich richtige und die Inanspruchnahme von Hilfe erleichternde Berichterstattung in den Medien notwendig. Bislang fehlen jedoch Studien, die sich mit der Darstellung von Burnout in deutschen Printmedien vor allem im Hinblick auf die von Bahlmann et al. $[20,21]$ aufgeworfene Fragestellung zu den Krankheitsvorstellungen über Burnout und Depression beschäftigen. Vor diesem Hintergrund verfolgt diese Arbeit das Ziel, Überschneidungen und Differenzen zu identifizieren, die sich in der Berichterstattung über Burnout im Hinblick auf das psychiatrische Störungsbild der Depression zeigen.

\section{Methoden}

Um sich dem Phänomen Burnout in den Medien mithilfe einer Inhaltsanalyse zu nähern, wurden Tageszeitungen herangezogen, die nach Hasebrink [24] „das größte Potenzial für eine Meinungs- und Einstellungsbildung auf der zentralen Verarbeitungsroute sowie für Prozesse der Themensetzung “ bieten, zumal deren Onlineformate für eine noch breitere Streuung der Inhalte sorgen. Vor dem Hintergrund ihrer weiten Verbreitung, hoher Verkaufszahlen [25] und der Zugänglichkeit zu ihrem archivierten Material bilden die 3 überregionalen Tageszeitungen Bild, Süddeutsche Zeitung und Die Welt aus den Jahren 2016 und 2017 im Printformat die Stichprobe. Die Sonntagsausgaben der Zeitungen Bild (Bild am Sonntag) und Die Welt (Welt am Sonntag) wurden genauso in die Analyse miteinbezogen wie zusätzliche Printbeilagen aller 3 Zeitungen. Zur Filterung aller Textpassagen, die sich mit dem Thema Burnout beschäftigen, wurde das 
> Tab.1 Ausschnitt der Zusammenfassung (OK: Oberkategorie, UK: Unterkategorie).

\begin{tabular}{|c|c|c|c|}
\hline Artikelausschnitt & Paraphrase & Generalisierung & Kategorie \\
\hline $\begin{array}{l}\text { Aber auch individuelle Faktoren wie } \\
\text { Perfektionismus begünstigen ein } \\
\text { emotionales Ausbrennen. } \\
\text { Welt, } 01.04 .16 \text { S. } 142\end{array}$ & $\begin{array}{l}\text { Perfektionismus begünstigt als } \\
\text { individueller Faktor ein emotionales } \\
\text { Ausbrennen. }\end{array}$ & $\begin{array}{l}\text { Perfektionismus stellt einen Risiko- } \\
\text { faktor für Burnout dar. }\end{array}$ & $\begin{array}{l}\text { OK } 3 \\
\text { Risikofaktoren }\end{array}$ \\
\hline $\begin{array}{l}\text { Hörsturz und Burnout seien mittlerweile } \\
\text { die Regel an Stadttheatern. } \\
\text { SZ, 04.05.16 S. } 5\end{array}$ & $\begin{array}{l}\text { Hörsturz und Burnout kommen bei } \\
\text { Schauspielern an Stadttheatern } \\
\text { mittlerweile häufig vor. }\end{array}$ & $\begin{array}{l}\text { Schauspieler erkranken häufig an } \\
\text { Burnout. }\end{array}$ & $\begin{array}{l}\text { OK } 5 \\
\text { Betroffene } \\
\text { Gruppen }\end{array}$ \\
\hline $\begin{array}{l}\text { Moorbaden macht glücklich } \\
\text { Kurkliniken besinnen sich auf das alte } \\
\text { europäische Heilmittel und setzen es } \\
\text { in der Therapie von Burnout-Patienten } \\
\text { erfolgreich ein } \\
\text { Welt, 17.09.16 S. } 41\end{array}$ & $\begin{array}{l}\text { Moorbaden macht glücklich und } \\
\text { wird in der Therapie von Burnout- } \\
\text { Patienten erfolgreich eingesetzt. }\end{array}$ & $\begin{array}{l}\text { Moorbaden ist eine erfolgreiche The- } \\
\text { rapiemethode für Burnout-Patienten. }\end{array}$ & $\begin{array}{l}\text { UK } 8.4 .1 \\
\text { Alternative } \\
\text { Methoden } \\
\text { (OK } 8 \\
\text { Therapie) }\end{array}$ \\
\hline $\begin{array}{l}\text { „Ich brach } 2007 \text { mit Erschöpfung und } \\
\text { Burnout zusammen. Schuld war vor } \\
\text { allem eines: Schlafmangel. } \\
\text { Bild, 19.07.16 S. } 1,7\end{array}$ & $\begin{array}{l}\text { Arianna Huffington brach } 2007 \text { mit } \\
\text { Erschöpfung und Burnout aufgrund } \\
\text { von Schlafmangel zusammen. }\end{array}$ & $\begin{array}{l}\text { Schlafmangel stellt eine Ursache für } \\
\text { Burnout dar. }\end{array}$ & $\begin{array}{l}\text { UK } 4.7 \\
\text { Schlafmangel } \\
\text { (OK } 4 \\
\text { Ursachen) }\end{array}$ \\
\hline $\begin{array}{l}\text { Ihr letzter Arbeitgeber forderte so viele } \\
\text { Arbeitsstunden von ihr, dass sie krank } \\
\text { wurde. Burnout, nach } 400 \text { Überstunden. } \\
\text { Welt, 16.10.17, S. } 27\end{array}$ & $\begin{array}{l}\text { Nachdem sie bei ihrem letzten } \\
\text { Arbeitgeber } 400 \text { Überstunden leisten } \\
\text { musste, erkrankte sie an Burnout. }\end{array}$ & $\begin{array}{l}\text { Überarbeitung stellt eine Ursache für } \\
\text { Burnout dar. }\end{array}$ & $\begin{array}{l}\text { UK } 4.1 .1 \\
\text { Überarbeitung/ } \\
\text { Überlastung } \\
\text { (OK } 4 \\
\text { Ursachen) }\end{array}$ \\
\hline
\end{tabular}

Zeitungsmaterial mithilfe des Begriffs „burn/burn*“ durchsucht. Die Trunkierung sollte sicherstellen, bei der Archivsuche der Tageszeitungen alle möglichen Schreibweisen des Begriffs Burnout (Burnout, Burn-out, Burn-Out, Burn out, Burn Out) abzudecken. Ungeachtet der Treffer, die keinen Bezug zum Thema Burnout aufwiesen und aussortiert wurden, ergaben sich insgesamt 392 zu analysierende Zeitungsartikel. Weitere 230 Treffer ließen sich in Bildunterschriften, Inhaltsverzeichnissen, sich wiederholenden Klinikanzeigen und im Fernsehprogramm finden, die aufgrund der begrenzten Aussagekraft in der weiterführenden Analyse nicht berücksichtigt wurden. 75\% der Artikel enthielten den Begriff Burnout einmal. In 17\% der Artikel kam der Begriff Burnout 2-mal vor. Zwei der insgesamt $392 \mathrm{Ar}$ tikel wiesen den Begriff Burnout mehr als 10-mal auf.

Als Auswertungsmethode des Archivmaterials diente die qualitative Inhaltsanalyse, welche sich durch ein systematisches, datenreduzierendes Vorgehen zur vergleichenden Analyse von bedeutungshaltigem Material auszeichnet [26]. Mayring [27] unterscheidet hierbei 3 Grundformen des qualitativen Interpretierens, von denen sich die Zusammenfassung als Verfahren mit einer induktiven Kategorienbildung am sinnvollsten erwiesen hatte, um einen möglichst unvoreingenommenen Blick auf das Zeitungsmaterial zu richten. In mehreren Schritten galt es, zunächst alle relevanten Textstellen aus dem Material herauszufiltern und diese danach zu paraphrasieren, mit dem Ziel, den inhaltstragenden Kern der Aussage auf einer möglichst einheitlichen Sprachebene zu extrahieren [27]. In einem nächsten Schritt wurden die gewonnenen Paraphrasen generalisiert, sodass Paraphrasen gleicher Aussage entstanden und nach ihrem Bedeutungsgehalt selektiert wurden [27]. Alle verbleibenden inhaltstragenden Paraphrasen wurden in einem letzten Schritt durch Bündelung und Integration inhaltlich zusammenhängender Paraphrasen reduziert und bildeten in dieser Form die einzelnen Kategorien des Kategoriensystems [27]. - Tab. 1 zeigt einen Ausschnitt aus der zusammenfassenden Inhaltsanalyse.

Während der Auswertung wurde das Kategoriensystem immer wieder kritisch hinterfragt und mehrfach überarbeitet, Kategorien mit verhältnismäßig vielen Zitaten wurden so in Unterkategorien differenziert. Ein Codierleitfaden wurde entwickelt, der das Kategoriensystem inklusive Kategoriendefinitionen, Ankerbeispielen, also besonders typische Textstellen, und Codierregeln enthält [27]. Insgesamt konnten 516 Zitate zum Thema Burnout aus dem Material herausgefiltert werden. Die Anzahl der Zitate pro Oberkategorie liegt zwischen 3 und 114.

\section{Ergebnisse}

Für die Identifikation von Gemeinsamkeiten und Differenzen in der Berichterstattung von Burnout im Hinblick auf das Störungsbild der Depression, lassen sich folgende Kategorien heranziehen: Ursachen, Symptome, therapeutische Maßnahmen, Personen des öffentlichen Lebens, Vergleich von Burnout und Depression und die Metaphorisierung des Begriffs Burnout.

\section{Ursachen}

Welche Ursachen einem Burnout zugrunde liegen, wird in den ausgewählten Printmedien vielschichtig betrachtet. Fast ein Viertel aller herausgefilterten Zeitungszitate beschäftigt sich mit diesem Aspekt. Neben Gründen wie enttäuschte Erwartungen (4\%), Stress (6\%), Rollenkonflikte (9\%), digitale Medien 
(4\%), Life Events (3\%), innere Ursachen (10\%) sowie Schlaf- (5\%) und Bewegungsmangel (3\%) stellen Arbeit und die fehlende Abgrenzung von Arbeit und arbeitsfreier Zeit die mit Abstand am häufigsten thematisierten Ursachen (44\%) dar. Eine entscheidende Rolle spielt dabei vor allem die Überarbeitung bzw. Überlastung: „Burnout gilt immer noch als Leiden, das durch Überarbeitung entsteht [...]." (Welt am Sonntag 04.12.16, S. 18) Aber auch der meist mit der Arbeit im Zusammenhang stehende Leistungsdruck kann laut Sven Hannawald, selbst von einem Burnout betroffen, dazu führen: „Viele denken, sie müssen immer nur funktionieren. [...] Wenn man immer nur funktioniert, macht es irgendwann peng." (SZ 23.09.16, S. 22) Dass sich auch die fehlende Abgrenzung von Arbeit und arbeitsfreier Zeit, z.B. durch ständige dienstliche Erreichbarkeit, zu einem Burnout kumulieren kann, illustriert dieses Zitat: „Bis irgendwann gar nicht mehr klar war, wo der Job aufhörte und die Freizeit anfing. Dass das gar nicht so klug war, merkten die meisten, als die ersten Freunde mit Burnout zu kämpfen hatten.“ (SZ-Beilage Jetzt 27.03.17, S. 16) In den meisten Fällen erweckt die mediale Darstellung den Eindruck, einem Burnout liege eine alleinige Ursache zugrunde. Nur in einem Beitrag wird betont, dass bei der Entstehung von Burnout mehrere Faktoren zugrunde liegen: „In der Regel führt eine Melange aus verschiedenen Lebensumständen zum Burnout." (SZ-Beilage wohl fühlen 06.04.16, S. 18-19) Keiner der Zeitungsartikel bringt biologische Faktoren im Zusammenhang mit der Entstehung von Burnout zur Sprache. Damit kommunizieren die ausgewählten Zeitungen ein grundlegend anderes Ursachenbild für einen Burnout als der wissenschaftliche Konsens mit einer Kombination aus psychosozialen und neurobiologischen Einflüssen für eine Depression.

\section{Symptome}

In der Darstellung der Symptome eines Burnouts, die in den Tageszeitungen 3,9\% der herausgefilterten Zitate ausmachen, dominieren mit Schlafstörungen, Herzbeschwerden, Schwindel und Tinnitus vor allem psychosomatische Symptome und mit Interessenverlust und Antriebslosigkeit Symptome, die einem Teil der Leitsymptome einer Depression entsprechen. Weitere Symptome wie Erschöpfung, Müdigkeit und der Verlust der Fähigkeit, abzuschalten, finden in den Zeitungen mehrfach $\mathrm{Er}$ wähnung, wenngleich die überwiegende Zahl von Symptomen nur einmalig benannt wird. Dass die Grenzen zwischen Burnout und Depression in der Mediendarstellung von Symptomen teilweise verschwimmen, illustriert auch das folgende Zitat aus der Bild-Zeitung vom 21.07.17 (S.6): „Erst kürzlich hatte er [Chester Bennington] BILD gegenüber von Depressionen und Alkoholproblemen gesprochen." Anschließend wird der Sänger selbst zitiert: „Das war eine echt harte Zeit. Ich hatte eine Art Burnout, aber nicht, weil ich mich müde fühlte. Ich hatte einfach die Schnauze voll von der Welt. Ich wollte nichts mehr machen, wollte niemanden sehen. Einmal habe ich meinem Therapeuten gesagt, dass ich nichts mehr hören kann. Ich war sogar so weit zu sagen, dass ich kein Mensch mehr sein wollte." Die Bild-Zeitung bezeichnet das Problem nach der Symptombeschreibung des Sängers also als Depression, der Sänger selbst als „eine Art Burnout“.

\section{Therapeutische Maßnahmen}

Therapeutische Maßnahmen finden in 8,7\% der analysierten Zitate Erwähnung. Darunter verdeutlichen 2 Textfragmente, dass Menschen mit Burnout professionelle Unterstützung benötigen. Zum größten Teil entstammen die dargestellten therapeutischen Mittel und Methoden jedoch dem alternativmedizinischen Spektrum (31\%), gefolgt von Bewegung und Sport (21\%), Ruhe und Erholung (10\%), Körperbewusstsein (10\%) und Onlinetherapie (10\%). Bevorzugte therapeutische Optionen zur Behandlung von Depressionen wie die Psycho- und Pharmakotherapie werden im Zusammenhang mit Burnout nur in einem Zitat benannt, sodass sich in der Printberichterstattung ein klarer Unterschied in den mit Burnout und Depressionen assoziierten therapeutischen Möglichkeiten zeigt.

\section{Personen des öffentlichen Lebens}

Als „Sprachrohr“ dienen Medien Personen des öffentlichen Lebens, wenn diese als Betroffene ihre Erfahrungen mit Burnout mit der Öffentlichkeit teilen: „Die Kommunikationswissenschaftlerin und Chefredakteurin der Wirtschaftswoche hatte 2010 ihre eigenen Burnout-Erfahrungen öffentlich gemacht.“ (SZ 25.04.16, S. 23) Ebenso Oliver Kahn „hat offen über einen Burnout in seiner Zeit bei Bayern München gesprochen“ (SZ 26.06.17, S. 8) und Fußballtrainer Sascha Lewandowski, der wegen eines Burnouts zurückgetreten war, „hatte darum gebeten, dass die Diagnose veröffentlicht wird“" (Bild 10.06.16, S. 1, 12).

\section{Vergleich mit Depression}

Dass Burnout nur in Ausnahmefällen als Tabuthema angesehen wird, könnte mit einem von den Medien beschriebenen geringeren gesellschaftlichen Stigma von Burnout im Vergleich zu Depressionen assoziiert sein: „Psychische Erkrankungen sind nach wie vor ein Stigma: Man gesteht sich ja heutzutage keine Depression mehr ein, sondern nennt es Burnout." (SZ 27.01.16, S. 2) Etwas bildlicher pointiert diesen Unterschied ein anderer Artikel: „Bei ,Depression“ mag so mancher an einen Menschen denken, der im dreckigen Unterhemd auf dem Sofa liegt; beim ,Burnout' taucht vor dem inneren Auge eher ein smarter Workaholic auf - in einer Leistungsgesellschaft eindeutig das attraktivere Bild. Wer ein Burnout hat, hat zu heftig gebrannt; wer depressiv ist, ist zu schwach, um je gebrannt zu haben." (SZ 20.08.16, S.49) Oftmals als „Leiden der Tüchtigen“ (Welt 01.04.16, S. 142) bezeichnet, wird Burnout im Gegensatz zu Depressionen mit Stärke und Erfolg verknüpft. Die Süddeutsche Zeitung schreibt in einem Artikel vom 30.01.16: „Ausgebrannt zu sein ist gesellschaftlich anerkannt, zumal in einer Leistungsgesellschaft wie der deutschen. Wer über Dauerstress klagt, signalisiert Einsatz, Hingabe, Unentbehrlichkeit und kann sich einer gewissen Bewunderung seiner Mitmenschen sicher sein." Erfolg legitimiert also die Entwicklung eines Burnouts. In einem Interview berichtet die Autorin Iny Klocke: „Ich fühle mich langsam total ausgebrannt!“ Darauf die Bild-Zeitung: „Verständlich, nach 31 Romanen in 13 Jahren!“ (Bild am Sonntag 23.10.16, S.44). Ob das „ausgebrannt sein“ hier im pathologischen Sinn oder als Metapher begriffen wird, lässt sich nicht sicher ausmachen. 


\section{Metaphorisierung}

Häufig wird der Begriff Burnout in den analysierten Tageszeitungen in einem metaphorischen Kontext verwendet, der weder einen medizinischen noch wissenschaftlichen Bezug erkennen lässt. In einem Großteil der Beiträge kommt der Begriff Burnout zur Sprache, wenn etwas nicht mehr funktioniert bzw. in einer Krise steckt: „Wäre die Mode eine Person, würde sie sich gerade einen Aufenthalt in der Burnout-Klinik buchen." (SZ Magazin Stil leben 20.10.16, S. 10-12) Einige Zitate lassen noch eine Steigerung des „Nichtfunktionierens“ erkennen: Die westlichen Demokratien befänden sich in einem „Zustand des Burnout“ (Welt 09.06.16, S. 8) und es wird davor gewarnt, dass „die Menschheit [...] die Erde in einen lebensbedrohlichen Burnout [treibt]“ (Bild 27.10.16, S. 10). Hier wird der Begriff eher im Sinne von ,dem Scheitern nahe“ oder ,zugrunde gehen“ verwendet. In einem Beitrag der Welt am Sonntag vom 17.01.16 über einen Film, in dem „man alles über den Burnout einer Liebe erfährt“, wird der Begriff sogar stellvertretend für die Wörter ,Scheitern', ,Ende‘ bzw. ,Aus‘ gebraucht. Die vergleichsweise häufige Verwendung des Begriffs Burnout in einem metaphorischen Kontext durch die Printmedien lässt erahnen, dass dieser entkoppelt von Medizin und Wissenschaft Einzug in die Alltagssprache gehalten hat und damit an Nähe gewinnt.

\section{Diskussion}

Die Ergebnisse der qualitativen Inhaltsanalyse von den 3 überregionalen Tageszeitungen Bild, Süddeutsche Zeitung und Die Welt aus den Jahren 2016 und 2017 zeigen, dass Burnout nach wie vor ein präsentes Thema in deutschen Printmedien ist - dafür sprechen die Anzahl und die thematische Vielfalt der herausgefilterten Zitate zum Thema. Das vielgestaltige Bild von Burnout zeigt sich jedoch nur in der Zusammenschau verschiedener Artikel aller 3 Zeitungen. Nimmt man an, dass für die meisten Menschen die mehr oder weniger regelmäßige Rezeption einer einzelnen Zeitung üblich ist, bekämen diese so eine eher einseitige Sicht auf das Phänomen Burnout. Zudem fällt in $75 \%$ der Artikel das Wort ,Burnout' nur einmalig und zumeist eher beiläufig. Nur wenige Zeitungsartikel beschäftigen sich explizit mit dem Thema, nur 2 Artikel der Welt vom 01.04.16 und 04.12.16 weisen unter dem Suchbegriff ,Burnout' 10 oder mehr Treffer auf. Zusammen mit den inhaltlichen Befunden der Analyse weist dies darauf hin, dass sich in wenigen Artikeln eine differenzierte, wissenschaftlich orientierte Darstellung des Themas findet. Dieser Befund korrespondiert auch mit den Ergebnissen einer inhaltsanalytischen Untersuchung von Hoffmann-Richter [28] zu (Vor-)Urteilen gegenüber psychiatrischen Themen in den Printmedien. Darin wird beschrieben, dass die häufige Nennung von psychiatrischen Begriffen wie Schizophrenie, Psychopharmaka und Psychotherapie in den Zeitungen zunächst vermuten lässt, dass das Thema in der Mitte der Gesellschaft angekommen ist. Doch eine tiefergehende Analyse offenbart, dass diese Begriffe inhaltlich meist nicht ausgeführt werden und der ausführlichen Darstellung von wissen- schaftlichen Erkenntnissen - und damit der Wissensvermittlung - kaum Platz eingeräumt wird.

Die identifizierten Überschneidungen und Differenzen zur Depression in der Berichterstattung von Burnout lassen Parallelen zu den Aussagen der Allgemeinbevölkerung in den Arbeiten von Bahlmann et al. [20, 21, 29] erkennen. Als die mit Abstand am häufigsten in den Tageszeitungen thematisierten Ursachen für Burnout kristallisierten sich Arbeit und die fehlende Abgrenzung von Arbeit und arbeitsfreier Zeit heraus, während biologische Ursachen oder Korrelate nicht beschrieben werden. Ebenso sieht die Allgemeinbevölkerung eine Depression eher als vererbt an und bringt Burnout eher mit beruflichen Belastungen in Verbindung. Auch Golla [30] zeigt in einer qualitativen Inhaltsanalyse der Darstellung von Depression in Printmedien, dass hier die biologischen vor den psychosozialen Ursachen deutlich dominieren. Die Ausführungen zu Symptomen von Burnout in den Tageszeitungen offenbaren in der Zusammenschau ein eher diffuses Bild aus einer Reihe von psychosomatischen Symptomen und einem Teil der Leitsymptome einer Depression. Ohne sich damit fundiert auseinanderzusetzen, bilden die Medien damit dennoch im Kleinen den wissenschaftlichen Diskurs über das Sammelsurium an Symptomen ab. Demnach existieren zahlreiche Burnout-Skalen, die insgesamt 130 unterschiedliche Symptome unter dem aktuellen Burnout-Konzept vereinen [17]. Die mediale Darstellung therapeutischer Maßnahmen bei Burnout zielt in großen Teilen auf Mittel und Methoden aus dem alternativmedizinischen Spektrum sowie Bewegung und Sport ab und ist vor allem vor dem Hintergrund der sich ähnelnden Symptomausprägung von Burnout und Depressionen als kritisch anzusehen. Bahlmann [20] und Golla [30] zeigen, dass Depressionen sowohl in der Bevölkerung als auch in den Medien ganz selbstverständlich mit stärkeren Empfehlungen von psychiatrischer, psychotherapeutischer und medikamentöser Hilfe assoziiert sind. Auch die Tatsache, dass lediglich an 2 Stellen Therapieempfehlungen für von Burnout Betroffene ausgesprochen werden, zeigt, dass Burnout eine andere Tragweite als Depressionen hinsichtlich der Krankheitsfolgen zu implizieren scheint.

Dass in den Tageszeitungen Personen des öffentlichen Lebens von ihren Erfahrungen als Betroffene berichten und der Begriff häufig als Metapher Verwendung findet, verdeutlicht, was die Auswertung der Kategorien Ursachen, Symptome und therapeutische Maßnahmen bereits andeuten: Während das Störungsbild der Depression auch aufgrund der möglichen genetischen Bedingtheit viel abstrakter und unbeeinflussbar wirkt und deshalb mit mehr Distanz betrachtet wird, wohnt dem Phänomen Burnout eine höhere soziale Toleranz inne [30]. Durch die Offenbarungen prominenter Betroffener, den Bezug zur Arbeit und die bildliche Qualität des mittlerweile in den (Sprach-)Alltag eingezogenen Begriffs ist jeder fähig sich etwas darunter vorzustellen - es entsteht Nähe. Dies kann den gesellschaftlichen Diskurs über das Thema Burnout erleichtern und wirkt sich so möglicherweise positiv auf das Verständnis und die Akzeptanz gegenüber Betroffenen sowie deren Hilfesuchverhalten aus. 
Mit dem Wissen um das große Wirkpotenzial der Medien im Meinungsbildungsprozess stellt sich die Frage, ob die nahbare Schilderung des Phänomens Burnout umgekehrt die Distanz zum Phänomen Depression vergrößert. Trendstudien zu den Einstellungen der Öffentlichkeit gegenüber Menschen mit psychischen Krankheiten zeigen, dass insbesondere Menschen mit schweren psychischen Störungen heute stärker abgelehnt werden als vor 20-30 Jahren [22]. Im ungünstigsten Fall könnte eine Entstigmatisierung von Befindlichkeitsstörungen einer stärkeren Stigmatisierung schwerer psychischer Krankheiten Vorschub leisten [31, 32] - eine wichtige Forschungsfrage, die beispielsweise in experimentellen Studien untersucht werden könnte.

\section{Stärken und Limitationen}

Trotz umfangreicher Berichterstattung und Forschung zu Burnout existiert aktuell keine Studie zur Darstellung von Burnout in deutschen Printmedien. Die vorliegende Untersuchung reagiert mit ersten Ergebnissen, die in Bezug zum Störungsbild Depression gesetzt werden, auf diese Forschungslücke. Eine qualitativ orientierte Untersuchung hat sich dafür als vorteilhaft erwiesen, da eine rein quantitative Analyse anhand bloßer Begriffshäufungen zu Verzerrungen hätte führen können. Die qualitative Analyse zeichnet ein vielschichtigeres Bild, das auch darüber Auskunft gibt, dass Begriffen und Erläuterungen häufig die wissenschaftliche Fundierung fehlt und die sich abzeichnende Differenzierung zwischen Burnout und Depression Stigmatisierungsprozesse forcieren könnte - 2 Aspekte, die verdeutlichen, wie wichtig es ist, die Darstellung von psychischen Störungen in den Printmedien kritisch zu verfolgen und zum Gegenstand der Forschung zu machen.

Die Ergebnisse sind aufgrund der Stichprobengröße nicht generalisierbar. Zudem wurde die mediale Darstellung von Burnout lediglich für einen engen zeitlichen Bereich untersucht und lässt keine Rückschlüsse über Veränderungen der Darstellungsweise im zeitlichen Verlauf zu. Die Methode der qualitativen Inhaltsanalyse ist aufgrund ihrer Regelgebundenheit für andere zwar nachvollziehbar, dennoch bietet die Auswertung des Materials stellenweise Interpretationsspielraum.

\section{KONSEQUENZEN FÜR KLINIK UND PRAXIS}

- Gemeinsamkeiten zeigen sich in der Symptomatik von Burnout, die Bezüge zu den Leitsymptomen einer Depression aufweist.

- Differenzen werden in Ursachen und therapeutischen Maßnahmen sichtbar. Das Phänomen Burnout wirkt durch Offenbarungen prominenter Betroffener, den Arbeitsbezug und die bildliche Qualität des Begriffs nahbarer als eine Depression.

- Zukünftige Forschung könnte klären, inwiefern eine Entstigmatisierung von Befindlichkeitsstörungen eine stärkere Stigmatisierung von schwereren psychischen Krankheiten möglicherweise fördert.
Interessenkonflikt

Die Autorinnen/Autoren geben an, dass kein Interessenkonflikt besteht.

Literatur

[1] Ärzteblatt. Weltgesundheitsversammlung beschließt die ICD-11. Im Internet: (Stand: 15.10.2019) https://www.aerzteblatt.de/nachrichten/103394/Weltgesundheitsversammlung-beschliesst-die-ICD-11

[2] Zeit Online. Burn-out erstmals als Krankheit anerkannt. Im Internet: (Stand: 15.10.2019) https://www.zeit.de/gesellschaft/2019-05/gesundheit-burnout-who-krankheiten-transgender

[3] Bild. WHO erkennt Burnout als Krankheit an. Im Internet: (Stand: 15.10.2019) https://www.bild.de/ratgeber/gesundheit/gesundheit/ erschoepfung-weniger-leistung-who-erkennt-burnout-als-krankheitan-62234174.bild.html

[4] Welt. WHO erkennt Burn-out als Krankheit an. Im Internet: (Stand: 15.10.2019) https://www.welt.de/gesundheit/article194300605/ WHO-erkennt-Burn-out-erstmals-als-Krankheit-an.html

[5] WHO. ICD-11 for Mortality and Morbidity Statistics. QD85 Burnout (04/2019). Im Internet: (Stand: 16.10.2019) http://id.who.int/icd/ entity/129180281

[6] Bonfadelli H, Friemel TN. Medienwirkungsforschung. 6. Aufl., Band Nr. 3451. UTB Soziologie, Psychologie, Pädagogik. Konstanz, München: UVK; 2017

[7] Hasebrink U. Macht als Wirkungspotenzial. Zur Bedeutung der Medienwirkungsforschung für die Bestimmung vorherrschender Meinungsmacht. Medien-Kurzanalysen. Berlin: Friedrich-Ebert-Stiftung; 2009

[8] WHO. Burn-out an „occupational phenomenon“: International Classification of Diseases. (28.05.2019). Im Internet: (Stand: 16.10.2019) https://www.who.int/mental_health/evidence/burn-out/en/

[9] Bundesvereinigung der Deutschen Arbeitgeberverbände. Burnout weiter keine Krankheit. Stellungnahme zum Burnout-Syndrom und zur ICD-11 Definition. Im Internet: (Stand: 15.10.2019) https://arbeitgeber.de/www/arbeitgeber.nsf/res/CE4733343CE644FCC1258 4650044CA22/\$file/Stn-Burnout-Syndrom.pdf

[10] Brooks M. Burnout Inclusion in ICD-11: Media Got It Wrong, WHO Says. Im Internet: (Stand: 15.10.2019) https://www.medscape. com/viewarticle/914077

[11] Gerhard S. Darum ist Burnout keine Krankheit. Im Internet: (Stand: 15.10.2019) https://www.quarks.de/gesellschaft/psychologie/darum-ist-burnout-keine-krankheit/

[12] Spiegel Online. WHO definiert Burn-out erstmals als Syndrom. Im Internet: (Stand: 15.10.2019) https://www.spiegel.de/gesundheit/diagnose/burn-out-durch-arbeitsbelastung-von-who-erstmals-als-syndrom-definiert-a-1269543.html

[13] Wolf C. Warum Burnout keine Krankheit ist. Im Internet: (Stand: 16.10.2019) https://www.spektrum.de/news/warum-burnout-keinekrankheit-ist/1653764

[14] Scherrmann U. „Burnout“: Begriff - Definition - Terminologie und Diagnose. In: Scherrmann U, Hrsg. Erste Hilfe bei Burnout in Organisationen. Ein Ratgeber für Führungskräfte und Personalverantwortliche. Wiesbaden: Springer; 2017: 3-7

[15] Thalhammer M, Paulitsch K. Burnout - eine sinnvolle Diagnose? Kritische Überlegungen zu einem populären Begriff. Neuropsychiatrie: Klinik, Diagnostik, Therapie und Rehabilitation: Organ der Gesellschaft Österreichischer Nervenarzte und Psychiater 2014; 28: 151159 
[16] Koch U, Broich K. Das Burn-out-Syndrom. Bundesgesundheitsblatt Gesundheitsforschung - Gesundheitsschutz 2012; 55: 161-163

[17] Burisch M. Das Burnout-Syndrom. Theorie der inneren Erschöpfung. 4. Aufl. Berlin: Springer; 2010

[18] Schaufeli WB, Enzmann D. The burnout companion to study and practice: A critical analysis. Issues in occupational health. London: Taylor \& Francis; 1998

[19] Härter M, Schneider F. Affektive Störungen (F3). In: Schneider F, Hrsg. Facharztwissen Psychiatrie, Psychosomatik und Psychotherapie. 2. Aufl. Berlin, Heidelberg: Springer; 2017: 337-363

[20] Bahlmann J, Angermeyer MC, Schomerus G. „Burnout“ statt „Depression“ - eine Strategie zur Vermeidung von Stigma? Psychiat Prax 2013; 40: 78-82

[21] Bahlmann J, Schomerus G, Angermeyer MC. Nicht ganz dasselbe: Krankheitsvorstellungen von Burnout und Depression in der Allgemeinbevölkerung. Psychiat Prax 2015; 42: 443-447

[22] Angermeyer MC, Matschinger H, Schomerus G. Attitudes towards psychiatric treatment and people with mental illness: changes over two decades. Br J Psychiatry 2013; 203: 146-151

[23] Pupato K. Psychiatrie in den Medien. In: Gaebel W, Baumann A, Hrsg. Stigma - Diskriminierung - Bewältigung. Der Umgang mit sozialer Ausgrenzung psychisch Kranker. Stuttgart: Kohlhammer; 2005: 8399

[24] Hasebrink U. Meinungsbildung und Kontrolle der Medien. (09.12.2016). Im Internet: (Stand: 08.10.2019) http://www.bpb.de/ gesellschaft/medien-und-sport/medienpolitik/172240/meinungsbildung-und-kontrolle-der-medien? $p=$ all
[25] Informationsgemeinschaft zur Feststellung der Verbreitung von Werbeträgern e. V. Quartalsauflagen. Im Internet: (Stand: 26.01.2019) https://www.ivw.eu/aw/print/qa

[26] Schreier M. Qualitative Analyseverfahren. In: Hussy W, Schreier M, Echterhoff G, Hrsg. Forschungsmethoden in Psychologie und Sozialwissenschaften für Bachelor. Springer-Lehrbuch. 2. Aufl. Berlin, Heidelberg: Springer; 2013: 245-275

[27] Mayring P. Qualitative Inhaltsanalyse. Grundlagen und Techniken. 11. Aufl., Beltz Pädagogik. Weinheim: Beltz; 2010

[28] Hoffmann-Richter U. Psychiatrie in der Zeitung: Urteile und Vorurteile. Bonn: Ed. Das Narrenschiff im Psychiatrie-Verl; 2000

[29] Bahlmann J, Schomerus G, Angermeyer MC et al. Macht Burnout Menschen ärgerlicher als Depression? Unterschiede in den emotionalen Reaktionen auf psychische Probleme in der Allgemeinbevölkerung. Zeitschrift für Psychiatrie, Psychologie und Psychotherapie 2016; 64: 19-24

[30] Golla BR. Darstellung der Depression in deutschen Printmedien 1999-2009 [Dissertation]. Greifswald: Ernst-Moritz-Arndt-Universität; 2018

[31] Peter O, Lang J, Stein K et al. „Ich bin nicht die Diagnose - aber sie ist auch ein Teil von mir" - Eine qualitative Interviewstudie zu Sichtweisen von psychisch erkrankten Menschen. Psychiat Prax 2019; 46: 2733

[32] Fegert JM, Baumeister H, Brieger P et al. Greifswalder Erklärung zur gesellschaftlichen Bedeutung des Bereichs psychische Gesundheit in der Gesundheitsforschung - „Lost in Translation?“ Psychiat Prax 2019; 46: 70-72 